\title{
Career pathways
}

\section{High quality patient care can only be based \\ upon a foundation of sound education and training, monitored by robust peer} assessment

David Barnard and Malcolm Pendlebury The Royal College of Surgeons of England
A $s$ we enter a new century and a new millennium never before has there been such a range of career pathways available to members of the dental profession. Whilst the opportunities for individual members of the profession are to be welcomed, some concerns have been expressed that the new arrangements for specialist training disadvantage practitioners who, having progressed as generalists, may wish to train as specialists later in their career.

We firmly believe that high quality patient care can only be based upon a foundation of sound education and training, monitored by robust peer assessment. This must be supported by a commitment to continuing professional development and lifelong learning - a principle which has recently been underpinned by the formal launch of the arrangements for recertification by the General Dental Council.

The medical Royal Colleges and their Faculties have traditionally set and monitored standards within the profession since the first sitting of the examination for the Licence in Dental Surgery was held at The Royal College of Surgeons of England in 1860. The Faculty of Dental Surgery (FDS) was established in 1947 to advance the science and the art of dental surgery and to encourage study and research. The Fellowship in Dental Surgery was established in 1948, and was followed by a range of specialist diplomas specific to defined clinical areas of dentistry. The Diploma of Membership in General Dental Surgery, a qualification specifically designed for GDPs, was first offered in 1979. The responsibility for this examination passed to the Faculty of General Dental Practitioners (FGDP(UK)) on its establishment in 1992.

Both the FDS and the FGDP(UK) provide educational programmes to support lifelong learning and continuing professional education and development for their fellows, members, associates and trainees. Both Faculties are committed to the principles of general professional training to provide broad based training and experience in various disciplines of dentistry, supported by sound educational programmes. The key to this must be flexibility and recent proposals from the Faculties will introduce further options for career development and address recent concerns.

While entry to specialist training remains the MFDS Diploma, it has been proposed that exemption from Parts $\mathrm{A}$ and $\mathrm{B}$ of the examination together with the requirement to undertake a minimum of one year in approved training posts, will be available to experienced practitioners who hold a diploma of the FGDP(UK) and can provide evidence of significant continuing professional education in accordance with an agreed protocol. This will make formal specialist training, leading to entry to a specialist list, more accessible to the general practitioner, who at a later stage in their career may decide to make a career change. Conversely, holders of the MFDS Diploma will be exempt from MGDS Part 1 to encourage entry to a career pathway in general practice for those who elected to gain the MFDS diploma following qualification. Reciprocity continues between MFGDP Part 1 and MFDS Part A and there is a commitment to continue to work towards a convergence of training requirements and a diploma to be taken towards the end of the period of general professional training.

The Dental Faculties remain committed to promoting and advancing the highest standards of care both within general practice and specialist practice. Considerable effort has been made to reconcile this primary responsibility to the public and to the profession with the provision of realistic and flexible training opportunities for the individual practitioner. 